\title{
De la educación a la orientación hacia la sociedad: hacia una profesionalización de la gestión de comunicación en Latinoamérica
}

\author{
From education to orientation towards society: \\ towards a professionalization of communication \\ management in Latin America
}

\section{Da educação à orientação para a sociedade: para uma profissionalização da gestão da comunicação na América Latina}

\author{
Cristina Navarro ${ }^{1}$ \\ Gulf University for Science y Technology (Kuwait) \\ cristina.navarro@urjc.es \\ Ángeles Moreno ${ }^{2}$ \\ Universidad Rey Juan Carlos (España) \\ mariaangeles.moreno@urjc.es
}

Fecha de recepción: 06 de febrero de 2018

Fecha de recepción evaluador: 30 de septiembre de 2018

Fecha de recepción corrección: 06 de noviembre de 2018

\footnotetext{
${ }^{1}$ Cristina Navarro es doctora en Comunicación por la Universidad Rey Juan Carlos y profesora asistente en Gulf University for Science and Technology. Es miembro del Grupo de Estudios Avanzados en Comunicación y autora de diversas publicaciones. https://orcid.org/0000-0003-0522-4428

2 Ángeles Moreno es doctora con premio extraordinario por la Universidad Pontificia de Salamanca y Profesora Titular en la Facultad de Comunicación de la Universidad Rey Juan Carlos. Ha enseñado e impartido cursos en diversas instituciones educativas. https://orcid.org/0000-0002-0777-2957
} 


\title{
Resumen
}

Esta investigación longitudinal cuantitativa analiza la evolución de la profesionalización de las relaciones públicas en América Latina utilizando datos procedentes de dos encuestas realizadas en los años 2009 y 2015. En total, 1.477 profesionales participaron en estos dos estudios, confirmando la idea de que la profesionalización es un proceso dinámico (Pieczka y L'Etang, 2001, 2006). Los resultados mostraron pequeñas pero significativas mejoras en cuatro indicadores: el estudio formal de las relaciones públicas en instituciones de educación superior, la existencia de unos principios éticos que hacen hincapié en el bienestar de la sociedad, el peso de las organizaciones profesionales, la disponibilidad de un cuerpo formal del conocimiento, y el acceso al empleo y la promoción. En general, se aprecia que los profesionales más jóvenes tienden a ser más críticos con respecto a los diferentes factores de profesionalización.

Palabras clave: profesionalización, relaciones públicas, América Latina, educación.

\begin{abstract}
This quantitative longitudinal research uses two datasets from 2009 and 2015 to assess the evolution of professionalization of public relations in Latin America. In total, 1,447 professionals participated in the studies, supporting the idea that professionalization is a dynamic process (Pieczka y L'Etang, 2001, 2006). Findings showed significant, but small improvements in four indicators: formal education, ideology and ethical principles, professional associations, and formal body of knowledge. In general, younger professionals tend to be more critical about the different factors of professionalization.
\end{abstract}

Keywords: professionalization, public relations, Latin America, education.

\section{Resumo}

Esta pesquisa longitudinal quantitativa utiliza dois conjuntos de dados de 2009 e 2015 para avaliar a evolução da profissionalização das relações públicas na América Latina. No total, 1.447 profissionais participaram dos estudos, apoiando a ideia de que a profissionalização é um processo dinâmico (Pieczka y L'Etang, 2001, 2006). Os resultados mostraram melhorias significativas, mas pequenas, em quatro indicadores: educação formal, ideologia e princípios éticos, associações profissionais e corpo formal de conhecimento. Em geral, os profissionais mais jovens tendem a ser mais críticos sobre os diferentes fatores da profissionalização.

Palavras-chave: profissionalização, relações públicas, América Latina, educação. 


\section{Introducción}

La profesionalización de la disciplina de relaciones públicas es un proceso de carácter dinámico (Pieczka y L'Etang, 2001, 2006), por lo que resulta interesante llevar a cabo seguimientos periódicos y continuados para evaluar su desarrollo en el tiempo. A medida que las relaciones públicas se han desarrollado en todo el mundo, la profesión ha ido acumulando normas y reglas que guían su práctica moderna y que dependen de las condiciones políticas, históricas, económicas, geográficas, demográficas y culturales de cada país (ej. Abdullah y Threadgold, 2008; De Bussy y Wolf, 2009; Dolea, 2012; Jeffrey y Brunton, 2012; Li, Cropp, Sims, y Jin, 2012; Molleda, Moreno, Athaydes, y Suárez, 2012; Park, 2003; Sriramesh y Verčič, 2009). La evolución de la disciplina afecta, a su vez, a las condiciones laborales de los profesionales que desarrollan su actividad en este campo de la comunicación (Niemann-Struweg y Meintjes, 2008).

América Latina es un continente geográfica y culturalmente diverso que constituye el $64 \%$ de la población del hemisferio occidental, aunque representa una parte relativamente pequeña de la economía mundial (Reyes y Sawyer, 2016). La región ha afrontado durante las últimas décadas desafíos socioeconómicos y políticos que han influido en el desarrollo de las relaciones públicas y obligado a los profesionales a buscar estrategias para poder posicionarse con éxito dentro y fuera de sus organizaciones.

El interés por las relaciones públicas en América Latina ha ido aumentando a lo largo de los últimos años como consecuencia de la mejoría económica en la región, los efectos de la globalización y el fortalecimiento del sistema de medios de comunicación. Reflejo de este renovado interés son los numerosos artículos publicados sobre la situación de las relaciones públicas en diferentes países de América Latina (ej., Álvarez, Yaguache y Paladines, 2016; De Brooks, Peñaloza, y Waymer, 2009; Mellado y Barría, 2012; Molleda, Athaydes y Hirsch, 2003; Molleda y Moreno, 2006, 2008; Molleda y Suárez, 2005). Sin embargo, la Macroencuesta Latinoamericana de Comunicación y Relaciones Públicas (Molleda, Moreno, Athaydes y Suárez, 2012) fue el primer intento de investigar el profesionalismo en el subcontinente recabando las opiniones de una muestra representativa de profesionales. Desde 2015, el Latin American Communication Monitor (Moreno, Molleda, Athaydes y Suárez, 2015, 2017), vinculado a estudios similares en Europa y Asia, permite comparar las prácticas y los desafíos de los comunicadores latinoamericanos con los del resto del mundo.

A pesar del creciente número de publicaciones sobre este campo de estudio, es importante seguir documentando de forma sistemática la práctica en diversas partes del mundo, especialmente en regiones poco representadas, como es el caso de América Latina. Este estudio tiene un doble objetivo: a) analizar los niveles de profesionalización de las relaciones públicas en América Latina y b) comprobar de qué manera han evolucionado los diferentes factores que influyen en este proceso en el periodo 20092015 . 


\section{Marco Teórico}

El crecimiento de la profesión de relaciones públicas y su apertura a nuevas disciplinas llegó de la mano de la internacionalización de los mercados y la globalización económica (Gold, Rogers, y Smith, 2002; Meintjes y Niemann-Struweg, 2009). Sin embargo, la industria continúa enfrentándose a problemas y complicaciones que influyen en su proceso de profesionalización.

Los conceptos de profesionalización y profesionalismo se usan a menudo de forma intercambiable erróneamente. El profesionalismo se relaciona con la formación continua y con el desarrollo de los profesionales con el objetivo de mejorar su empleabilidad (Chung y Choi, 2012; Niemann-Struweg y Meintjes, 2008). Sin embargo, la profesionalización se entiende como el proceso llevado a cabo por una organización o por una industria con el objetivo de alcanzar unos niveles adecuados y generalmente aceptados de calidad y excelencia.

Según Wilensky (1964), para alcanzar el estatus profesional, cualquier ocupación debe poner en marcha escuelas de capacitación, formar asociaciones profesionales, regular la práctica a través de la protección legal y adoptar un código ético. Nessmann (1995) también identifica la educación, las asociaciones profesionales y los códigos éticos como pasos fundamentales hacia la profesionalización.

\section{El concepto de profesionalización en relaciones públicas}

Profesionalización es un constructo que, pese a los esfuerzos realizados por muchos investigadores del campo de las relaciones públicas, aún adolece de una conceptualización adecuada. Las dos perspectivas teóricas más influyentes en el área de la sociología de las profesiones son la teoría de los rasgos -dominante durante gran parte del siglo XX-y la del poder (o poder/conflicto), enfoque que surgió en la década de 1970 para superar las deficiencias de la anterior. Los defensores del enfoque fenomenológico o de los rasgos se centran en la articulación de un conjunto de atributos básicos que todas las profesiones pueden compartir. McLeod y sus colegas siguieron esta tradición en las décadas de los 60 y 70 del siglo pasado y propusieron un índice que ha sido aplicado a varias ocupaciones relacionadas con la comunicación, incluidas las relaciones públicas (Bissland y Rentner, 1989; Hallahan, 1974; Nayman, McKee, y Lattimore, 1977; Wright, 1979).

Cameron, Sallot y Lariscy (1996) revisaron la literatura académica sobre la profesionalización de las relaciones públicas y concluyeron que solo cuatro artículos publicados hasta ese momento utilizaban una base empírica que permitiera avanzar en los intentos de definir este fenómeno y posteriormente evaluar su progresión. En concreto se referían a la labor de Gitter y Jaspers (1982), Ryan (1986), Judd (1989), y Rentner y Bissland (1990).

En los últimos años, los investigadores han abordado la profesionalización como un constructo multidimensional (David, 2004; Lages y Simkin, 2003). En este sentido, Meyer y Leonard (2014: 377) afirman que "la descripción de los requisitos para la profesionalización [...] parece reflejar una mayor conciencia de la conexión entre el 
campo de la gestión de la comunicación y su impacto en la sociedad", y hacen énfasis en la ética para avanzar en su efectiva autorregulación.

Aunque se han realizado numerosos intentos para consensuar los parámetros que permitan evaluar la profesionalización de las relaciones públicas, todavía no se llegado a un verdadero acuerdo (Cameron, Sallot, y Weaver-Lariscy, 1996; Cutlip, Center, y Broom, 1994; J. E. Grunig y Hunt, 1984; Niemann-Struweg y Meintjies, 2008; Parsons, 2008). Entre la variedad de marcos propuestos, los cuatro indicadores principales de profesionalización más utilizados son: la educación y la formación continua estandarizada, el código deontológico común, la existencia de asociaciones profesionales, y el seguimiento de procesos de acreditación estructurados.

El PRSA Professional Bond Report (Commission on Public Relations Education, 2006) incluyó factores adicionales como, por ejemplo, el sentido de responsabilidad, la organización de actividades globales y el propósito de trabajar por una sociedad mejor. Pese a que el interés por la profesionalización ha decaído en popularidad, esta área de estudio sigue siendo importante para avanzar hacia el reconocimiento que merece la disciplina (Evetts, 2014). La presente investigación operacionalizó los pasos a la profesionalización en ocho categorías:

1. Educación. La estandarización de la educación es uno de los indicadores de profesionalización más utilizados en las relaciones públicas (Meyer y Leonard, 2014; Nessmann, 1995; Yang y Taylor, 2013). Disponer de un plan de estudios común permite unificar el conocimiento de la profesión que se imparte en las instituciones de enseñanza superior, y asegura que las mejores prácticas se transmitan a los futuros profesionales. La formación continua se refiere, sin embargo, al desarrollo de los profesionales que ya forman parte de la industria y permite ofrecer respuestas a los nuevos retos de una sociedad en continua evolución, actualizando sus prácticas y técnicas.

2. Ética. Los códigos éticos otorgan respeto, credibilidad y legitimidad (J. E. Grunig, 2000, Meyer y Leonard, 2014; Yang y Taylor, 2013). Este tipo de normas deontológicas proporcionan consistencia a la profesión, animando a los profesionales y a las organizaciones a regular su toma de decisiones siguiendo normas de procedimiento compartidas por todos (Taylor y Yang, 2013).

3. Asociaciones profesionales. El establecimiento de una asociación profesional proporciona estabilidad, facilita la rendición de cuentas y aumenta los recursos disponibles para los profesionales (J. E. Grunig, 2000; Nessmann, 1995; Yang y Taylor, 2013, 2014). Por otra parte, una asociación sirve para facilitar la transparencia y denunciar prácticas poco éticas (Yang y Taylor, 2013).

4. Corpus formal de conocimiento. El término profesionalización se usa a menudo en el campo de las relaciones públicas para referirse a las prácticas basadas en la investigación científica producida en las universidades (Meintjes y NiemannStruweg, 2009). El conocimiento formal está íntimamente asociado con el auge de la ciencia moderna y la aplicación del método científico a los problemas sociales (Freidson, 1988). 
5. Técnicas especificas desdinadas al mercado de trabajo. La profesionalización, como proyecto colectivo, necesita de conocimientos específicos, incluyendo técnicas y habilidades (Larson \& Larson, 1979). Este conocimiento está vinculado a los valores y necesidades centrales del sistema social y ofrece estatus y carácter distintivo a la profesión. Van Ruler (2005) señala que, a nivel académico, la existencia de un conjunto de conocimientos y habilidades predefinidos es un camino hacia la profesionalización.

6. Trabajo especializado. Para que una ocupación evolucione al rango de profesión requiere de trabajadores que desempeñen labores que no estaban previamente cubiertas por otros profesionales. Además, debe existir un control de la mano de obra especializada, tanto para garantizar una calidad de servicio como para mejorar la situación del propio profesional (Morris, Crawford, Hodgson, Shepherd y Thomas, 2006). Aunque la gama de actividades que realizan las relaciones públicas es amplia, el trabajo exige dos componentes principales: la adquisición de técnicas de comunicación y capacidad de asesorar a la gerencia para lograr los objetivos de la organización (Johansen, 2001).

7. Prácticas diferenciadas. La profesionalización de las relaciones públicas requiere de prácticas diferenciadas. Aunque comparte teorías con otros campos de conocimiento como la comunicación de masas, la comunicación interpersonal, la psicología (social), o la economía, Hundhausen fue capaz de definir en 1974 los contornos académicos de las relaciones públicas (Bourne, 2015; Ihlen y van Ruler, 2007).

8. Un sistema de acceso y promoción del empleo es otro indicador clave. La estandarización y formalización de los procedimientos de selección, retención y desarrollo profesional han mejorado el proceso de profesionalización, evitando el nepotismo y el clientelismo (Evetts, 2014). Una mayor transparencia también favorece las opciones de carrera.

Para poder llevar a cabo el presente trabajo, y con el objetivo de analizar el nivel y la evolución de la profesionalización en América Latina, se formularon las siguientes preguntas de investigación:

PI1: ¿Cuáles son los indicadores de profesionalización que fueron más valorados por los profesionales de las relaciones públicas de América Latina en 2015 ?

PI2. ¿Cuáles son las diferencias estadísticamente significativas entre los datos recogidos sobre la profesionalización de las relaciones públicas en 2009 y 2015 ?

\section{Metodología}

Molleda, Moreno, Athaydes y Suárez (2010) contribuyeron al debate sobre la profesionalización con un estudio realizado en 2009 en el que evaluaron los niveles de profesionalización de las relaciones públicas en América Latina y probaron un índice diseñado a partir de la obra de los sociólogos de las profesiones Freidson $(1983,2001)$ y Krause (1996). Usando la misma escala de profesionalización, Molleda, Kochhar y 
Moreno (2012) conceptualizaron y probaron un modelo de profesionalización de las relaciones públicas y de los roles sociales que desempeñan los profesionales en sus organizaciones. Los puntos fuertes de la escala de profesionalización, así como la necesidad de observar la evolución de las relaciones públicas en América Latina, son las bases del presente estudio longitudinal comparativo.

\section{Encuesta y demográficos}

El estudio de 2015 empleó información procedente del Latin American Communication Monitor, una encuesta realizada por ocho universidades de España, Estados Unidos y América Latina en cooperación con asociaciones profesionales de la región. Los datos se recogieron mediante una encuesta online diseñada en Qualtrics, traducida al español y al portugués, y que se mantuvo activa entre los meses de octubre de 2014 y febrero de 2015. Una red de contactos personales, junto a las bases de datos de profesionales pertenecientes a diferentes asociaciones, facilitó la distribución de la encuesta. Las invitaciones para participar en el estudio fueron enviadas a casi 20.000 profesionales en 19 países. A pesar de que 1.774 comenzaron la encuesta, tan solo la terminaron 821 individuos. Las respuestas de los participantes que no pudieron ser identificados claramente como parte de la población de estudio se eliminaron del conjunto de datos. Esta selección estricta de los encuestados marca una diferencia clara con respecto a otros estudios que utilizan el muestreo de bola de nieve o aquellos que incluyen estudiantes, académicos y personas no ligadas directamente la profesión. La evaluación final se basó en 803 respuestas completas de profesionales de las relaciones públicas y gestión de la comunicación de América Latina.

Solo nueve países $(\mathrm{N}=749)$ reunieron el mínimo de respuestas requeridas por los investigadores para poder establecer comparaciones estadísticas: Argentina $(\mathrm{N}=118)$, Brasil $(\mathrm{N}=201)$, Chile $(\mathrm{N}=67)$, Colombia $(\mathrm{N}=139)$, Costa Rica $(\mathrm{N}=36)$, México $(\mathrm{N}$ $=68)$, Perú $(\mathrm{N}=36)$, República Dominicana $(\mathrm{N}=47)$, y Venezuela $(\mathrm{N}=31)$.

El perfil de los profesionales latinoamericanos descrito por la encuesta es urbano y joven, pero formado y experimentado. En torno a la mitad de los participantes son líderes de comunicación: el 23,9\% ocupan la máxima posición de comunicación o son CEO de una consultora y el $24,5 \%$ son directores de equipo o de un área de comunicación. E1 52,6\% de los profesionales lleva más de diez años trabajando en el ámbito de la gestión de comunicación o relaciones públicas y presenta un alto nivel educativo, destacando el $51,8 \%$ que posee un título de maestría. Asimismo, tres cuartas partes de los encuestados desempeñan su actividad principal en una de las tres primeras ciudades de su país.

La mayoría trabaja en empresas privadas (27,5\%); un 22,9\% trabaja en el sector público o en organizaciones políticas; el $20,5 \%$ en consultorías y agencias; el $12 \%$ en organizaciones sin ánimo de lucro y un 7,6\% por cuenta propia. Un dato sorprendente, debido a la creciente globalización de los mercados, es que solo un 2,4\% declara tener su actividad principal en el área de comunicación internacional y un 7,2\% de los participantes es miembro de alguna asociación internacional de comunicación. 
En cuanto a su origen, la gran mayoría de los encuestados $(67,7 \%)$ proceden de América del Sur (incluyendo Argentina, Bolivia, Brasil, Chile, Colombia, Ecuador, Perú, Uruguay y Venezuela). Una proporción menor de los encuestados (más del 24\%) trabaja en América Central y el Caribe (incluyendo Costa Rica, Cuba, El Salvador, Guatemala, Honduras, Nicaragua, Panamá y República Dominicana), y el 8,5\% en México.

Del mismo modo, la encuesta realizada en 2009 también se diseñó en Qualtrics y se distribuyó utilizando la red personal de colegas y las bases de datos de las asociaciones profesionales. Un total de 1.150 personas comenzaron encuesta y 674 la completaron. Solo diez países $(\mathrm{N}=612)$ cumplieron con el número mínimo de observaciones establecidas por los investigadores: Argentina $(\mathrm{N}=59)$, Brasil $(\mathrm{N}=102)$, Chile $(\mathrm{N}=38)$, Colombia $(\mathrm{N}=104)$, Costa Rica $(\mathrm{N}=67)$, Guatemala $(\mathrm{N}=39)$, México $(\mathrm{N}=80)$, Panamá $(\mathrm{N}=23)$, Perú $(\mathrm{N}=33)$, y Venezuela $(\mathrm{N}=67)$.

La edad promedio de los participantes fue de 38 años $(\mathrm{SD}=11.14)$. El $71 \%$ eran mujeres y el 39\% hombres. La mayor parte trabajaba en el sector privado (36\%), agencias y consultorías (26\%), el gobierno (24\%), organizaciones sin ánimo de lucro (7\%), y freelancers $(7 \%)$. El promedio de años de experiencia en relaciones públicas era de 12 $(\mathrm{SD}=9)$. Más de un tercio de los participantes contaba con un título universitario y un $13 \%$ de maestría. Más de la mitad de los participantes ocupaba la posición más alta del departamento de relaciones públicas o comunicación dentro de sus organizaciones. Casi el $50 \%$ de los encuestaos eran miembros de asociaciones profesionales y el $31 \%$ ocupaba posiciones de liderazgo.

\section{Resultados}

\section{Clasificación de los indicadores de Profesionalización en 2015}

Los tres indicadores de profesionalización que recibieron las mejores evaluaciones por parte de los profesionales latinoamericanos en 2015 fueron los que indican que las relaciones públicas y la gestión de la comunicación "se estudian formalmente en instituciones de educación superior que otorgan títulos o diplomas" (M $=4,25)$, "se basan en una ideología y principios éticos que implica explícitamente un compromiso con el bien de la sociedad" $(\mathrm{M}=3,63)$, y "se basan en un cuerpo formal del conocimiento (académico, científico)" $(\mathrm{M}=3,53)$. Por el contrario, los tres indicadores en la parte inferior de la escala de evaluación fueron los que afirman que las prácticas de relaciones públicas "tienen un sistema de acceso y promoción laboral en el que se priman más las habilidades y conocimientos profesionales que el nepotismo" $(\mathrm{M}=2,86)$, "se reconocen como un trabajo especializado dentro de las organizaciones" $(\mathrm{M}=3,19)$, y "es una práctica diferenciada de otros campos relacionados como el marketing, la publicidad y el periodismo" $(\mathrm{M}=3,27)$.

Los resultados obtenidos confirman que la edad es un factor de predicción de la evaluación de la profesionalización. En particular, la gran mayoría de los indicadores se relacionan con la edad de los participantes: la ideología y la base ética, el estatus especial en el mercado, la existencia de organizaciones profesionales, la práctica diferenciada, el cuerpo formal de conocimiento, el trabajo especializado en las organizaciones, y el 
sistema de acceso y promoción del empleo. Es decir, a mayor edad, mejor evaluación de los indicadores de profesionalización.

\section{Evaluación comparativa de los resultados de 2009 y 2015}

Para comparar y contrastar los dos conjuntos de datos se utilizó una prueba $t$ de muestras independientes. La comparación 2009-2015 de cinco de los ocho indicadores de profesionalización fue estadísticamente significativa. De este modo, en 2015 los participantes evaluaron mejor la enseñanza de las relaciones públicas en las instituciones de educación superior, los principios ideológicos y éticos, la existencia de asociaciones, el conocimiento científico formal, y el sistema de promoción y acceso al empleo. La evaluación del resto de los indicadores se mantuvo igual entre los dos periodos de tiempo.

Las diferencias por país también fueron notables. Venezuela, Costa Rica y Perú experimentaron el mayor progreso en el proceso de profesionalización. Por el contrario, Argentina, Colombia y México apenas sufren cambios significativos. La Tabla 1 muestra los estadísticos descriptivos de los ocho indicadores para 2009 y 2015.

Tabla 1. Estadística descriptiva comparativa 2009-2015

Las relaciones públicas y la gestión de comunicación ...

\begin{tabular}{llll}
\multicolumn{2}{c}{ 2009 } & \multicolumn{2}{c}{$\mathbf{2 0 1 5}$} \\
$\mathbf{N}$ & $\mathbf{M}$ & \multicolumn{1}{l}{$\mathbf{N}$} & $\mathbf{M}$ \\
668 & 3,89 & 803 & 4,25 \\
& & & \\
666 & 3,44 & 803 & 3,63 \\
667 & 3,19 & 803 & 3,53 \\
666 & 3,23 & 803 & 3,39 \\
& & & \\
659 & 3,31 & 803 & 3,37 \\
& & & \\
665 & 3,21 & 803 & 3,27 \\
669 & 3,18 & 803 & 3.19 \\
664 & 3,04 & 803 & 2,86 \\
\hline
\end{tabular}

... Se estudian formalmente en instituciones de educación superior que otorgan títulos o diplomas

... Se basan en una ideología y principios éticos que implica explícitamente un compromiso con el bien de la sociedad.

... Se basan en un cuerpo de conocimiento formal (académico, científico)

... Cuentan con organizaciones asociativas que agrupan a los profesionales para su beneficio.

... Poseen conocimientos y técnicas propios que otorgan a los profesionales un estatus especial en el mercado de trabajo.

... Es una práctica diferenciada de otros campos relacionados como el marketing, la publicidad y el periodismo.

... Se reconocen como un trabajo especializado dentro de las organizaciones.

... Tienen un sistema de acceso y promoción laboral en el que se priman más las habilidades y conocimientos profesionales que el nepotismo (amiguismo o influencia personal) 


\section{Discusión y conclusiones}

Este estudio apoya la idea de que la profesionalización de las relaciones públicas y la gestión de la comunicación es un proceso dinámico. En 2015, los profesionales de relaciones públicas de América Latina evaluaron altamente tres indicadores de profesionalización (la educación formal, la ideología y los principios éticos para el bien de la sociedad, y el cuerpo académico/científico de conocimiento). Sin embargo, fueron menos optimistas con respecto a la especialización de las relaciones públicas en las organizaciones y en el mercado, así como con la existencia de asociaciones profesionales que trabajen en beneficio de los profesionales del sector. Esta investigación también describe la lucha continua de las relaciones públicas para lograr y mantener su legitimidad (Merkelsen, 2011). En general, se aprecia que los profesionales más jóvenes tienden a ser más críticos respecto a los diferentes factores de profesionalización. Esto puede representar un obstáculo añadido a los esfuerzos de legitimación e institucionalización llevados a cabo por los defensores de las relaciones públicas.

La comparación de datos de 2009 y 2015 ofrece claras evidencias de que las relaciones públicas en América Latina es una profesión u ocupación en proceso de cambio. Cinco de los ocho indicadores de profesionalización experimentaron cambios significativos, aunque pequeños. Los participantes indicaron avances positivos en el estudio formal de las relaciones públicas en instituciones de educación superior, la existencia de unos principios éticos que hacen hincapié en el bienestar de la sociedad, en el peso de las organizaciones profesionales, la disponibilidad de un cuerpo formal del conocimiento, además de en el acceso al empleo y la promoción. A pesar del pequeño cambio positivo, las asociaciones de relaciones públicas en América Latina parecen tener dificultades para lograr una representación significativa de las comunidades profesionales en la mayoría de los países. También existe cierta preocupación por la falta de acceso al empleo y a las promociones basadas en méritos y habilidades. El clientelismo, el nepotismo, el amiguismo o la influencia personal siguen siendo importantes a la hora de desarrollarse profesionalmente y alcanzar puestos de responsabilidad. Esta puede ser una de las razones que impulsan a los jóvenes profesionales a mostrarse más críticos con los niveles de profesionalización de las relaciones públicas.

La evaluación de otros indicadores de profesionalización se mantuvo prácticamente estática en los dos períodos de tiempo (es decir, su estatus especial en el mercado de trabajo, especialización dentro de las organizaciones y el hecho de ser una práctica diferenciada de otros campos relacionados como el marketing, la publicidad y el periodismo). Este dato resulta preocupante, especialmente en un momento en que las tecnologías están borrando las líneas que separan las diferentes disciplinas relacionadas con la comunicación.

\section{Implicaciones teóricas y prácticas}

Este estudio ofrece documentación adicional y pruebas evidentes de la naturaleza dinámica de la profesionalización de las relaciones públicas y la gestión de la comunicación. El enfoque comparativo y longitudinal utilizado en el análisis de dos conjuntos de datos se realiza con poca frecuencia en los estudios de relaciones públicas, 
más aún en determinadas regiones del mundo. Por primera vez se ofrece un estudio empírico longitudinal con estándares estadísticos sobre la evolución de la profesionalización en Latinoamérica.

Las comunidades profesionales y académicos deben invertir en el análisis, la defensa y la mejora de los indicadores de la profesionalización de las relaciones públicas en todo el mundo. A pesar de que Europa Occidental y América del Norte siguen siendo los principales mercados, los países emergentes necesitan avanzar en la consolidación de la gestión de la comunicación, una profesión que está hoy en día en el centro de los procesos de globalización y digitalización de datos. Las relaciones públicas en América Latina presentan indicios de evolución, institucionalización y profesionalización que deben tenerse en cuenta, especialmente aquellos ligados a su orientación hacia la sociedad.

\section{Limitaciones e investigación futura}

El enfoque de este estudio y su análisis s aborda una sección del Latin American Communication Monitor. La fatiga de los participantes puede haber afectado negativamente la calidad de los datos. Aunque se contactó una gran muestra de profesionales, un número muy pequeño inició y completó la encuesta en línea, tanto en 2009 como en 2015. Esto motivó la falta de representación de algunos países del continente. En el futuro, se necesita una mayor participación para permitir un análisis comparativo más completo.

\section{Referencias}

Abdullah, Z., y Threadgold, T. (2008). Towards the professionalization of public relations in Malaysia: Perception management and strategy development. Public Relations Review, 34(3), 285-287.

Nobell, A. A., Yaguache, J. J., y Paladines, F. G. (2016). Public Relations in Ecuador: Exploratory and Descriptive Research About Career Development. In New Advances in Information Systems and Technologies (pp. 761-770). Springer, Cham.

Bissland, J.H, y Rentner, T.L. (1989). Public relations' quest for professionalism: An empirical study. Public Relations Review, 15(3), 53.

Botan, C. (1992). International public relations: Critique and reformulation. Public Relations Review, 18, 149-159.

Bourne, C. D. (2015). Extending PR's critical conversations with advertising and marketing. The Routledge Handbook of Critical Public Relations, 119.

Brooks, K. P., y Waymer, D. (2009). Public relations and strategic issues management challenges in Venezuela: A discourse analysis of Crystallex International Corporation in Las Cristinas. Public Relations Review, 35(1), 31-39. 
Burns, E. (2007). Positioning a post-professional approach to studying professions. New Zealand Sociology, 22(1), 69-98.

Cameron, G. T., Sallot, L. M., y Lariscy, W. (1996). Developing standards of professional performance in public relations. Public Relations Review, 22(1), 43-61.

Chung, W., y Choi, J. (2012). Professionalism in public relations pedagogy: A comparative analysis of public relations curricula among the United States, the United Kingdom, and South Korea. Journalism y Mass Communication Educator, 67 (4), 375-391.

Commission on Public Relations Education. (2006). The Professional Bond: Public relations education and the practice. Retrieved from, http://www.commpred.org/_uploads/report2-executiveSummary.pdf

Coombs, W.T., Holladay, S., Hasenauer, G., y Signitzer, B. (1994). A comparative analysis of international public relations: Identification and interpretation of similarities and differences between professionalization in Austria, Norway, and the United States. Journal of Public Relations, 6(1), 23-39.

Culbertson, H.M. (1996). Introduction. In H.M. Culbertson y N. Chen (Eds). International public relations. A comparative analysis (pp. 1-13). Mahwah, NJ: Lawrence Erlbaum Associates.

Culbertson, H.M., Jeffers, D.W., Stone, D.B., y Terrell, M. (1993). Social, political, and economic contexts in public relations: theory and cases. Mahwah, NJ: Lawrence Erlbaum Associates.

Culbertson, H.M., y Jeffers, D.W. (1992). Social, political, and economic contexts: Key in educating true public relations professionals. Public Relations Review, 18, 5365 .

Curtin, P. A. (2012). Public relations and philosophy: Parsing paradigms. Public Relations Inquiry, 1(1), 31-47.

Cutlip, S. M., Center, A. H., y Broom, G. M. (1994). Effective Public Relations (7th ed.). Englewood Cliffs, NJ: Prentice Hall

David, P. (2004). Extending symmetry: Toward a convergence of professionalism, practice, and pragmatics in public relations. Journal of Public Relations Research, $16(2), 185-211$.

De Bussy, N. M., y Wolf, K. (2009). The state of Australian public relations: Professionalization and paradox. Public Relations Review, 35(4), 376-381.

Dolea, A. (2012). Status and Trends in Public Relations in Romania. EUPRERA Congress. Istanbul, Turkey, 20 - 22 September 2012.

Economy. (2015). Organisation for Economic Co-operation and Development website. Retrieved from: http://www.oecd.org/economy/ 
Edwards, L. (2012). Defining the 'object' of public relations research: A new starting point. Public Relations Inquiry, 1(1), 7-30.

Evetts, J. (2014). The concept of professionalism: Professional work, professional practice and learning. In International Handbook of Research in Professional and Practice-based Learning (pp. 29-56). Springer Netherlands.

Freidson, E. (1988). Professional powers: A study of the institutionalization of formal knowledge. University of Chicago Press.

Freidson, E. (2001). Professionalism: The third logic. Chicago, IL: University of Chicago Press.

Freidson, E. (1983). The theory of professions: State of the art. In R. Dingwall and O. Lewis (Eds.), The sociology of the profession: Lawyers, doctors and others (pp. 19-37). London: The Macmillan Press.

Gitter, A.G., y Jaspers, E. (1982). Are PR counselors trusted professionals? Public Relations Quarterly, 27, 28-31.

Gold, J., Rodgers, H., y Smith, V. (2002). The future of the professions: Are they up for it? Foresight, 4(2), 46-53.

Grunig, J. E. (2000). Collectivism, collaboration, and societal corporatism as core professional values in public relations. Journal of Public Relations Research. 12, 23-48.

Grunig, J. E., y Hunt, T. (1984). Managing public relations. New York: Holt, Rinehart, y Winston.

Hallahan, K.E. (1974). Professional association membership, professional socialization and the professional orientation of the public relations practitioner. Unpublished master's thesis, University of Wisconsin, Madison, WS.

Jeffrey, L., y Brunton, M. (2012). Professional identity: How communication management practitioners identify with their industry. Public Relations Review, $38(1), 156-158$.

Johansen, P. (2001). Professionalisation, building respectability, and the birth of the Canadian Public Relations Society. Journalism Studies, 2(1), 55-71

Judd, L.R. (1989). Credibility, public relations and social responsibility. Public Relations Review, 15(2), 34-40.

Krause, E. A. (1996). Death of the guilds: Professions, states, and the advance of capitalism, 1930 to the present. New Haven, CT: Yale University Press.

Lages, C., y Simkin, L. (2003). The dynamics of public relations: key constructs and the drive for professionalism at the practitioner, consultancy and industry levels. European Journal of Marketing, 37(1/2), 298-328. 
Larson, M. S., \& Larson, M. S. (1979). The rise of professionalism: A sociological analysis (Vol. 233). University of California Press.

L'Etang, J., y Pieczka, M. (Eds.). (2006). Public relations: Critical debates and contemporary practice. Mahwah, NJ: Laurence Erlbaum Associates.

Li, C., Cropp, F., Sims, W., y Jin, Y. (2012). Perceived professional standards and roles of public relations in China: Through the lens of Chinese public relations practitioners. Public Relations Review, 38(5), 704-710.

McLeod, J.M., y Hawley, S.E., Jr. (1964). Professionalization among newsmen. Journalism Quarterly, 46, 529-539.

Meintjes, C., y Niemann-Struweg, I. (2009). The role of a professional body in professionalisation: The South African public relations case. PRism, 6, 1-14.

Mellado, C., y Barría, S. (2012). Development of professional roles in the practice of public relations in Chile. Public Relations Review, 38(3), 446-453.

Merkelsen, H. (2011). The double-edged sword of legitimacy in public relations. Journal of Communication Management, 15(2), 125-143.

Meyer, A.L., y Leonard, A. (2014). Are we there yet? En route to professionalism. Public Relations Review, 40(2), 375-386.

Molleda, J. C., Athaydes, A., y Hirsch, V. (2003). Public relations in Brazil: Practice and education in a South American context. Global Public Relations Handbook: Theory, Research, and Practice. Mahwah, NJ: Lawrence Erlbaum, 356-77.

Molleda, J. C., Kochhar, S., y Moreno, A. (2012). An intertwined future: Exploring the relationship between the levels of professionalism and social roles of public relations practitioners. In M. W. Distaso (Ed.), Public Relations Society of America: Educators Academy (pp. 128-145). San Francisco: PRSA.

Molleda, J. C., y Moreno, Á. (2006). Transitional socioeconomic and political environments of public relations in Mexico. Public Relations Review, 32(2), 104109.

Molleda, J.C., y Moreno, A. (2008). Balancing public relations with socioeconomic and political environments in transition: comparative, contextualized research of Colombia, México and Venezuela. Journalism and Mass Communication Monographs, 10(2), 116-174.

Molleda, J. C., Moreno, Á., Athaydes, A., y Suárez, A. M. (2012). Macroencuesta latinoamericana de comunicación y relaciones públicas. Organicom, 7(13), 118141.

Moreno, A., Molleda, J. C., Athaydes, A., y Suárez, A. M. (2015). Latin American Communication Monitor 2014-2015. Excelencia en comunicación estratégica, 
trabajo en la era digital, social media y profesionalización. Resultados de una encuesta en 18 países. Recuperado de, http://latincommunicationmonitor.com.

Moreno, A., Molleda, J. C., Athaydes, A., y Suárez, A. M. (2017). Latin American Communication Monitor 2016-2017. Tendencias en comunicación estratégica: Big Data, automatización, engagement, influencers, coaching y competencias. Resultados de una encuesta en 17 países. Recuperado de, http://latincommunicationmonitor.com.

Molleda, J. C., y Suárez, A. M. (2005). Challenges in Colombia for public relations professionals: a qualitative assessment of the economic and political environments. Public Relations Review, 31(1), 21-29.

Morris, P. W., Crawford, L., Hodgson, D., Shepherd, M. M., \& Thomas, J. (2006). Exploring the role of formal bodies of knowledge in defining a profession-The case of project management. International Journal of Project Management, 24(8), $710-721$.

Nayman, O. McKee, B.K. y Lattimore, D.L. (1977). PR personnel and print journalists: A comparison of professionalism. Journalism Quarterly, 54(3) 492-497.

Nessmann, K. (1995). Public relations in Europe: A comparison with the United States. Public Relations Review. 21(2), 151-160

Niemann-Struweg, I., y Meintjes, C. (2008). The professionalism debate in South African public relations. Public Relations Review, 34(3), 224-229.

Park, J. (2003). Discrepancy between Korean government and corporate practitioners regarding professional standards in public relations: A co-orientation approach. Journal of Public Relations Research, 15(3), 249-275.

Parsons, P. (2008). Ethics in public relations: A guide to best practice. Kogan Page Publishers.

Pieczka, M., y L'Etang, J. (2006). Public relations and the question of professionalism. In J. L'Etang y M. Pieczka (Eds.), Public relations: Critical debates and contemporary practice (pp. 265-278). Mahwah, NJ: Laurence Erlbaum Associates.

Pieczka, M., y L'Etang, J. (2001). Public relations and the question of professionalism. In R. L. Heath (Ed.), Handbook of public relations (pp. 223-235). Thousand Oaks, CA: Sage Publications.

Rentner, T.L., y Bissland, J.H. (1990). Job satisfaction and its correlates among public relations workers. Journalism Quarterly, 67(4), 950-955.

Reyes, J. A., y Sawyer, W. C. (2016). Latin American economic development. New York: Routledge. 
Ryan, M. (1986). Public relations practitioners' views of corporate social responsibility. Journalism Quarterly, 63(4), 740-762.

Sriramesh, K., y Verčič, D. (2009). The global public relations handbook; Theory, research, and practice (Expended and revised edition). New York: Routledge.

Taylor, M. (2001). International public relations; opportunities and challenges for the $21^{\text {st }}$ century. In R.L. Heath (Ed.) y G. Vásquez (Contributing Ed.). Handbook of public relations (pp. 631-634). Thousand Oaks, CA: Sage.

The Holmes Report (2016). Global PR Industry Hits \$14bn In 2016 As Growth Slows To 5\%. Retrieved from: http://www.holmesreport.com/research/article/global-prindustry-hits-\$14bn-in-2016-as-growth-slows-to-5

Van Ruler, B. (2005). Commentary: Professionals are from Venus, scholars are from Mars. Public Relations Review, 31(2), 159-173.

Wilensky, H. (1964) The Professionalisation of Everyone? American Journal of Sociology 70(2), 137-58.

Wright, D. (1979). Professionalism and social responsibility in public relations. Public Relations Review, 5(3), 20-33.

Yang, A., y Taylor, M. (2014). A global perspective on public relations professionalism: Mapping the structure of public relations associations' international networks. Journalism and Mass Communication Quarterly, 91(3), 508-529.

Yang, A., y Taylor, M. (2013). The relationship between the professionalization of public relations, societal social capital and democracy: Evidence from a cross-national study. Public Relations Review, 39(4), 257-270.

\section{Notas}

1 Cristina Navarro es doctora en Comunicación por la Universidad Rey Juan Carlos (Madrid, España) y profesora asistente en Gulf University for Science and Technology (Kuwait). Además, es miembro del Grupo de Estudios Avanzados en Comunicación (GEAC) y autora de diversas publicaciones en revistas científicas nacionales e internacionales. Sus líneas de investigación abarcan la comunicación online, los canales social media, el liderazgo o la profesionalización del sector de las relaciones públicas. Anteriormente, trabajó durante casi dos décadas en diferentes medios de comunicación escritos y ha ejercido como consultora de agencias de relaciones públicas.

2 Ángeles Moreno es doctora con premio extraordinario por la Universidad Pontificia de Salamanca y Profesora Titular en la Facultad de Comunicación de la Universidad Rey Juan Carlos (Madrid). Ha enseñado e impartido cursos en diversas instituciones educativas en Europa y América. Es Directora Ejecutiva de la European Public Relations Research and Education Association, es miembro activo del Grupo de Estudios Avanzados en Comunicación (GEAC) y participa y dirige proyectos de investigación de la Unión Europea, la European Public Relations Research and Education Association (EUPRERA), el Plank Center de la Universidad de Alabama, la University of Florida y el MICINN. Forma parte desde hace diez años del equipo de investigación del mayor estudio sobre la Gestión de Comunicación en el mundo, el European Communication Monitor y es directora del proyecto Latin American Communication Monitor. Sus trabajos han sido premiados internacionalmente por la International Communication Association, la Public Relations Society of America y la European Public Relations and Research Association. 